\title{
The Basal Ganglia and Motor Control
}

\author{
Henk J. Groenewegen \\ Department of Anatomy, Research Institute Neurosciences Vrije Universiteit, VU University \\ Medical Center, Amsterdam, The Netherlands
}

\begin{abstract}
This paper briefly reviews the functional anatomy of the basal ganglia and their relationships with the thalamocortical system. The basal ganglia, including the striatum, pallidum, subthalamic nucleus, and substantia nigra, are involved in a number of parallel, functionally segregated cortical-subcortical circuits. These circuits support a wide range of sensorimotor, cognitive and emotionalmotivational brain functions. A main role of the basal ganglia is the learning and selection of the most appropriate motor or behavioral programs. The internal functional organization of the basal ganglia is very well suited for such selection mechanisms, both in development and in adulthood. The question of whether clumsiness may be, at least in part, attributed to dysfunction of the basal ganglia is discussed in the context of the differential, complementary, or interactive roles of the basal ganglia and the cerebellum in the development of motor control.
\end{abstract}

\section{KEYWORDS}

cerebellum, dopamine, development

Reprint requests to: H.J. Groenewegen $\mathrm{MD} \mathrm{PhD}$, Department of Anatomy, VU University Medical Center (VUmc), Van der Boechorststraat 7, 1081 BT Amsterdam, The Netherlands; e-mail: hj.groenewegen@vumc.nl

\section{INTRODUCTION}

Voluntary (intended) movements as well as unintentional movements are based on spatial and temporal patterns of muscle contractions that are initiated and coordinated by different structures in the central nervous system. The fine-tuning of these structures and the neuronal networks that are involved in motor execution are essential for the expression of adequate motor behavior. Most of our skilled movements, as well as our complex behaviors, have been learned in the course of development and have to be 'maintained' during adult life. Although in this extended, lifelong learning process extensive parts of the brain are important, in the case of skilled movements in particular the cerebral cortex, the cerebellum, and the basal ganglia have a crucial role. Clumsiness is a term associated in childhood with problems in the learning and execution of skillful movements, the neuronal basis of which is, however, poorly understood (Hadders-Algra, 2003). The knowledge of normal structural and functional relations among the brain structures involved in motor functions is essential for insight into the etiology and pathogenesis of various movement disorders, including clumsiness.

The execution of willed, intentional movements often requires the subtle, concerted action of the motor and sensory systems. Consider, for example, eye-hand coordination during the execution of fine manipulations with the hand and fingers. Complex movements have to be learned and they have to be practiced frequently. The correct execution of such 
movements is dependent upon the exact timing in the activation and switching of motor programs that have been imprinted in the brain during the course of motor learning processes (motor memory). In daily life, once a movement has been initiated, the execution of most of our willed, intentional movements occurs virtually automatically.

Intentional movements are in essence initiated by the cerebral motor cortex that directly, or indirectly via local premotor circuits, reaches the brain stem or spinal motor neurons that project to the muscles. Before a motor signal descends from the motor cortex to the brain stem and spinal cord, however, several cortical and subcortical centers, including the basal ganglia and the cerebellum, have posed their influence on the motor cortex to 'shape' the final, descending signal. The basal ganglia and the cerebellum exert their influence on the final motor output pathways largely via the thalamus on the descending, corticobulbar and corticospinal motor pathways that originate in the motor and premotor areas of the cerebral cortex. In this way, both the basal ganglia and the cerebellum have an essential and distinctive role in the organization (coordination, timing, and sequencing) of a normal motor output. Finally, the basal ganglia, as well as the cerebellum, play an important role in motor learning processes, albeit in different aspects and phases.

The present account briefly reviews the functional-anatomical aspects of the basal ganglia in relation to the brain circuits in which they are involved. The arrangement (described below) of 'intrinsic' connections between basal ganglia structures and their functional-anatomical relations with the (frontal) thalamocortical system, as well as with a number of centers in the mesencephalon, lend support to the hypothesis that the basal ganglia play a role in the facilitation of intended, desired motor programs and in the suppression of unintended or competing ones. The basal ganglia might also play a role in the learning of skillful movements and (complex) behavioral programs, both in early development as well as during later life. To what extent, if at all, clumsiness can be attributed to a malfunctioning of the basal ganglia or to disturbances that are related to a role of the basal ganglia in the development of normal motor behavior, remains an open question.

\section{STRUCTURE AND FIBER CONNECTIONS OF THE BASAL GANGLIA}

\section{Which structures belong to the basal ganglia?}

The brain structures that are included in the 'basal ganglia' consist of the striatum, the pallidum, the subthalamic nucleus, and the substantia nigra. Each individual structure is, in the human brain, constituted by macroscopically different subnuclei. Thus, the striatum includes the caudate nucleus, putamen, and nucleus accumbens, the pallidum consists of an internal and an external segment, and the ventral pallidum. The subthalamic nucleus appears, macroscopically, as an undivided morphological unity, but the substantia nigra has a clearly distinguishable pars compacta and pars reticulata. The ventral tegmental area (VTA), situated medially to the substantia nigra in the rostral mesencephalon, can also be considered a part of the basal ganglia 'family'.

The reason for including the caudate nucleus, putamen, and nucleus accumbens collectively in the striatum is that all three nuclei have similar histological, neurochemical, and connectional characteristics. The predominant striatal neuronal element is the medium-sized, densely spiny neuron that receives and integrates the bulk of striatal inputs from the cerebral cortex and thalamus and that projects to the pallidum and the substantia nigra (Fig. 1; Smith \& Bolam, 1990; Gerfen \& Wilson, 1996). Medium-sized spiny neurons contain the neurotransmitter gamma-amino butyric acid 
(GABA) and co-localize different neuropeptides. A minority of striatal neurons is formed by various types of interneurons, among which are cholinergic and parvalbuminergic, as well as a variety of peptidergic neurons (Bolam et al., 2000). Although the various parts of the striatum may transfer different types of information (on the basis of inputs from functionally distinct areas of the cerebral cortex; see below), as a result of its relative histological and neurochemical uniformity, the way in which these different types of information are 'processed' in the striatum is probably very similar. Likewise, distinct parts of the pallidal complex can transfer different types of information, but the morphological characteristics of the various subnuclei of the pallidal complex are comparable. Most of the pallidum consists of relatively large aspiny neurons that contain the neurotransmitter GABA; the neuronal density of the pallidum is much lower than that of the striatum. Pallidal neurons receive most of their input from the striatum and the subthalamic nucleus, and they project either to other basal ganglia structures or to the thalamus or the brain stem outside the basal ganglia circuitry (Gerfen \& Wilson, 1996)

In contrast to the striatum and the pallidum, the histological and connectional characteristics of the two parts of the substantia nigra are very different. In its histological, neurochemical, and

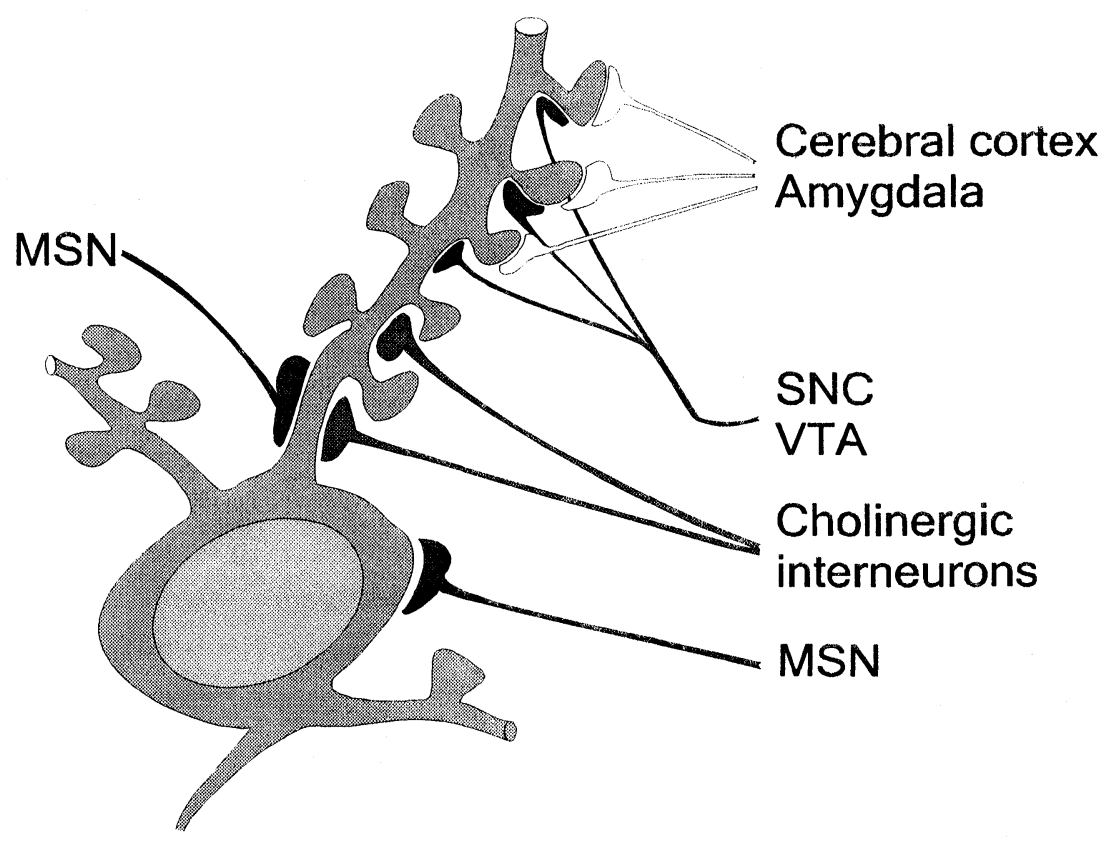

Fig. 1: Schematic representation of a medium-sized spiny neuron (MSN) in the striatum. The position of different inputs on the neuron are indicated: excitatory cortical or amygdaloid inputs end on the head of spines, dopaminergic terminals from the substantia nigra pars compacta (SNC) and ventral tegmental area (VTA) terminate in close association with the corticostriatal inputs on the spine necks or dendritic shafts. Inputs from neighboring medium-sized spiny neurons and striatal interneurons terminate on the proximal parts of the dendrites. Adapted from Smith and Bolam (1990). 
connectional characteristics, the pars reticulata most resembles the internal segment of the pallidum, while the pars compacta contains the dopaminergic neurons that project to both the striatum and the (pre)frontal cortex. The VTA contains both dopaminergic and GABAergic neurons in about equal quantities and can be considered a mixture of elements comparable with the pars compacta and pars reticulata of the substantia nigra. The subthalamic nucleus, situated at the junction between the ventral diencephalon and the mesencephalon, is a compact homogeneous group of neurons that use glutamate as neurotransmitter. The subthalamic nucleus is strongly influenced by inputs from the pallidum, as well as by extrinsic cortical and thalamic inputs. It sends glutamatergic projections to the pallidum and to the pars reticulata of the substantia nigra, exciting the output neurons of the basal ganglia (Gerfen \& Wilson, 1996; Wise et al., 1996).

\section{'Position' of the basal ganglia in forebrain circuits}

The striatum can be considered the main input structure of the basal ganglia in that the entire cerebral cortex, in a topographical manner, projects to the striatum while also the midline and intralaminar thalamic nuclei, the hippocampus and amygdala send fibers to the striatum. All these striatal inputs are excitatory (Parent \& Hazrati, 1995; Wise et al., 1996). The transfer of cortical and thalamic information through the striatum is modulated by dopaminergic and serotonergic inputs from the pars compacta of the substantia nigra and the mesencephalic raphe nuclei, respectively. The striatum is rich in dopamine D1 and D2 receptors, whereas various types of serotonergic receptors are expressed, among which the 5HT-2 receptors are most prominent (Gerfen \& Wilson, 1996). The ventral parts of the striatum, including the nucleus accumbens, as well as ventral parts of the caudate nucleus and putamen, receive 'limbic' inputs from the hippocampus and the amygdala; this part of the striatum contains the highest density of serotonergic receptors and expresses, in addition to the D1 and D2 receptors, the dopamine D3 receptor (Groenewegen et al., 1996; Diaz et al., 1995). The electrophysiological properties of medium-sized spiny output neurons of the striatum are such that they depend on convergent excitatory inputs (from the cerebral cortex and the thalamus) to become 'active'. In view of the very intricate patterns of overlap and segregation of inputs from (functionally) different cortical areas, this dependence most probably allows for a highly selective mechanism of activation of specific striatal regions or populations (ensembles) of striatal neurons, which is important for an understanding of the functions of the basal ganglia (Pennartz et al., 1994; Gerfen \& Wilson, 1996; see also below).

The main output of the basal ganglia is derived from the internal segment of the globus pallidus, the pars reticulata of the substantia nigra, and the ventral pallidum. These structures predominantly project to the ventral anterior and mediodorsal thalamic nuclei and reach in this way the cerebral cortical areas in the entire frontal lobe. Also, the centromedian-parafascicular thalamic nucleus is reached by pallidal inputs, and this thalamic structure projects to the motor cortex as well as to the striatum. In addition, pallidal and nigral outputs reach the superior colliculus, the mesencephalic reticular formation, and the pedunculopontine region, in this way influencing descending brain stem projections to the spinal motor apparatus (Parent \& Hazrati, 1995; Gerfen $\&$ Wilson, 1996).

The external segment of the globus pallidus and the subthalamic nucleus have very limited, if any, projections outside the basal ganglia circuitry but are intensively interconnected with each other, as well as with other subnuclei of the pallidal and nigral complex. These structures form part of the 
so-called 'indirect pathway', or rather 'indirect network' (Bolam et al., 2000), that is interposed between the striatum as the basal ganglia input structure and the output structures, namely the internal pallidal segment, ventral pallidum, and pars reticulata of the substantia nigra. A 'direct pathway' also exists between the basal ganglia input and output structures, which comprise the direct striatopallidal and striatonigral projections (Fig. 2; Gerfen \& Wilson, 1996). The prevailing

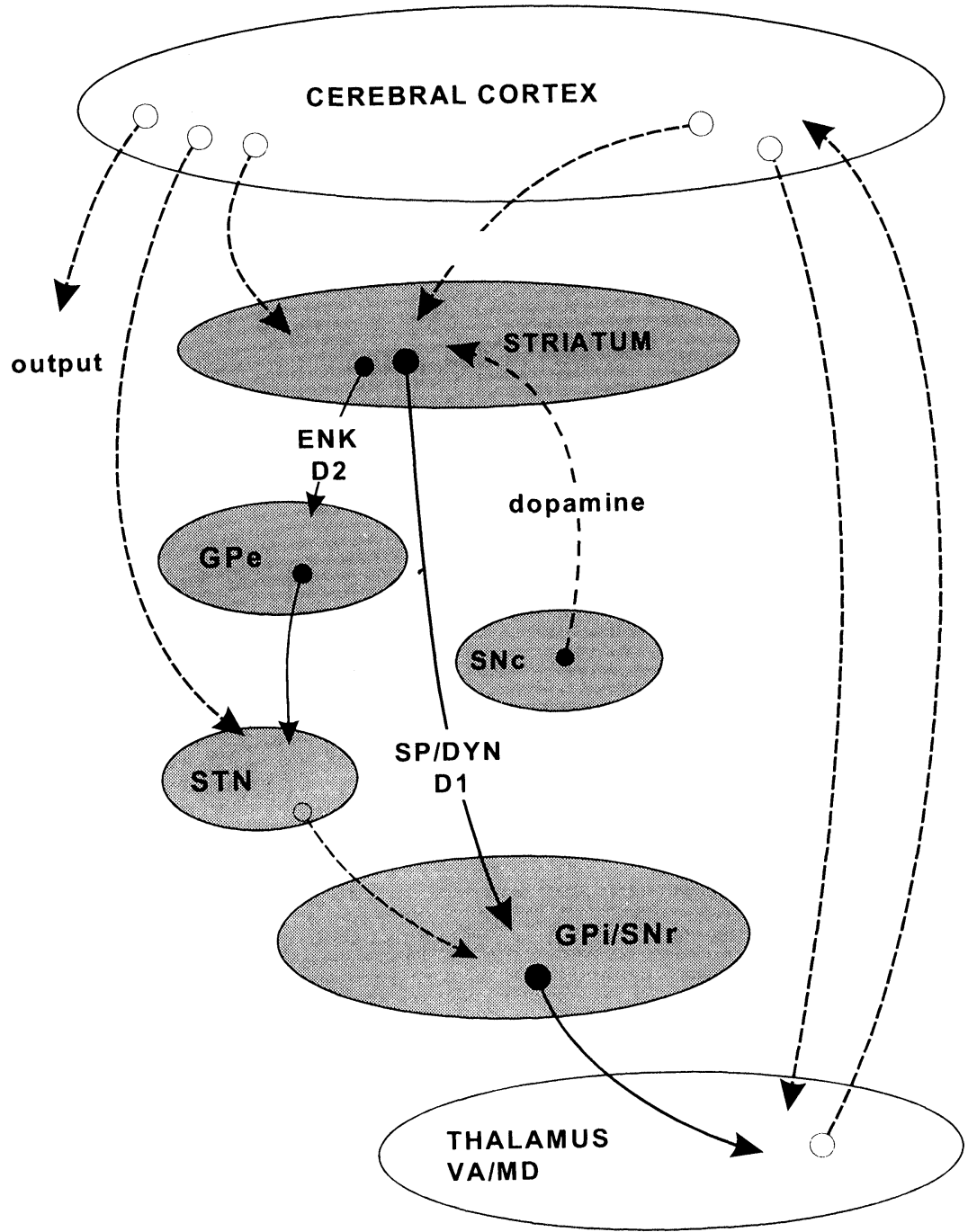

Fig. 2: Schematic representation of the organization of basal ganglia-thalamocortical circuits. Direct and indirect pathways between the striatum and the internal segment of the globus pallidus (GPi) and the substantia nigra pars reticulata $(\mathrm{SNr})$ are outlined. In the direct pathway the peptides substance $\mathrm{P}(\mathrm{SP})$ and dynorphin (DYN) are expressed, together with the dopamine D1 receptor. In the indirect pathway, the projection from the striatum to the external segment of the globus pallidus (GPe) contains the peptide enkephalin (ENK) and the dopamine D2 receptor. This indirect pathway is further constituted by the GABAergic projection from GPe to the subthalamic nucleus (STN) and the glutamatergic STN projections to GPi/SNr. In the thalamus the ventral anterior (VA) and mediodorsal (MD) nuclei are reached by the basal ganglia outputs. Solid lines representing projections code for GABAergic pathways, dashed lines for glutamatergic pathways, unless otherwise indicated. 
notion is that the direct and indirect pathways arise from two different populations of striatal mediumsized, spiny projection neurons. The direct pathway arises from striatal neurons that contain GABA, substance $\mathrm{P}$ and dynorphin as neurotransmitter/ neuromodulator and that express the dopamine D1 receptor. The striatal neurons that give rise to the indirect pathway, which reaches the external pallidal segment as a first way station in this multisynaptic pathway, contain GABA and enkephalin, and these neurons express the dopamine D2 receptor (Fig. 2; Gerfen \& Wilson, 1996). Subsequent projections in this indirect pathway include the GABAergic projections from the external segment of the globus pallidus to the subthalamic nucleus and, subsequently, the glutamatergic projections from the subthalamic nucleus to the internal segment of the globus pallidus and the pars reticulata of the substantia nigra (Fig. 2; Gerfen \& Wilson, 1996; Parent \& Hazrati, 1995).

The projection neurons in the basal ganglia output structures have the electrophysiological characteristic of being tonically active and in this way exert a tonic inhibitory influence on the thalamus and the mesencephalon. Interestingly, the direct and indirect striatal output pathways have opposing effects on the output neurons in the pallidum and the substantia nigra. Thus, activity in the direct striatal output pathway produces an inhibition of the tonically active pallidal and/or nigral output neurons, resulting in a disinhibition of their target areas (Chevalier \& Deniau, 1990). By contrast, a higher activity in the 'indirect network', for example through the activity of the indirect striatal output pathway, is 'translated' into an increased activity of the excitatory subthalamic projections to the basal ganglia outputneurons, leading to a stronger inhibition of the basal ganglia targets. If a higher activity in the (pre)frontal thalamocortical systems is considered to be associated with increased motor or cognitive/ behavioral output of the brain, we can conclude that the direct pathway facilitates, whereas the indirect pathway or network suppresses such output. Noteworthy is that the subthalamic nucleus not only receives a (tonic) inhibitory input from the external pallidal segment (and in this way is disinhibited during striatal activity) but also is projected upon directly by excitatory cortical and thalamic fibers (Gerfen \& Wilson, 1996; Feger et al., 1994). The cortical fibers originate mostly in the frontal cortex, whereas the thalamic fibers are derived from the centromedian-parafascicular complex. This means that (parts of) the cerebral cortex, as well as the caudal intralaminar thalamus play a role in a stronger inhibition of the basal ganglia target areas and, thereby, the suppression of motor and/or cognitive outputs.

Via different types of dopamine receptors in the two populations of striatal output neurons, dopamine has an opposing role on these output pathways of the striatum. Via the dopamine D1 receptor, the activity of the direct pathway is facilitated, whereas the dopamine D2 receptor suppresses the activity of the indirect pathway at the level of the basal ganglia output neurons (Gerfen \& Wilson, 1996). Therefore, higher striatal dopamine levels result in a disinhibition of the basal ganglia target areas, whereas lower dopamine concentrations at the striatal level lead to a stronger inhibition of the basal ganglia targets. The latter situation occurs in Parkinson's disease and is associated with bradykinesia and hypokinesia.

As indicated above, the projections from functionally different parts of the cerebral cortex to the striatum are topographically organized. The result of this organization is that the striatum can be subdivided into functionally different sectors, namely a sensorimotor sector receiving convergent inputs from motor, premotor, and sensory cortical areas; an associative sector receiving inputs from (pre)frontal, temporal, and parietal association cortical areas; and, finally, a 'limbic sector that is projected upon by the hippocampus, amygdala, 
and parahippocampal and orbitofrontal cortices (Fig. 3). Of course, no sharp boundaries exist between these functionally different striatal sectors. The sensorimotor sector is located dorsolaterally in the striatum (including dorsal parts of both the caudate and putamen), whereas in ventromedial direction, there is a gradual transition into the associative (including mainly the caudate nucleus, but also ventral parts of the putamen) and, subsequently, the 'limbic' sector of the striatum (including the nucleus accumbens and the most ventral parts of both caudate and putamen). The

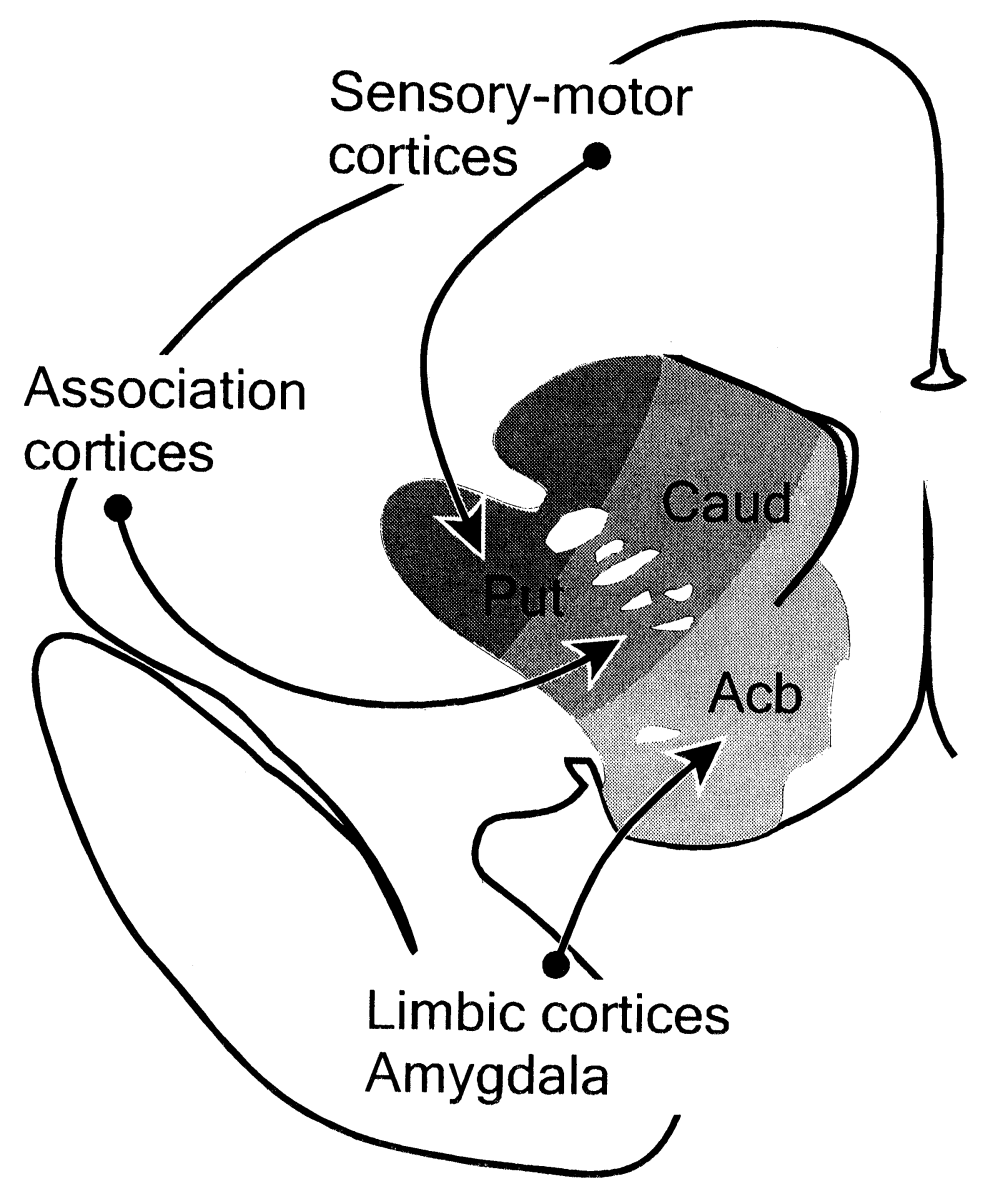

Fig. 3: Schematic representation of the topographic arrangement of the projections from functionally different cortical areas to the striatum, i.e., the caudate nucleus (Caud), putamen (Put), and the nucleus accumbens (Acb). The corticostriatal projections globally 'dictate' the functional subdivision of the striatum in a dorsolateral 'sensorimotor' part, an intermediate and medial 'cognitive' part and a ventrolateral 'emotional/motivational' part. Note that the functional zonation of the striatum which follows the corticostriatal organization does not comply with the macroscopic subdivision of the striatum in caudate nucleus, putamen and nucleus accumbens. 
subsequent projections from the striatum to the pallidum and substantia nigra, as well as the basal ganglia outputs to the thalamus, are also topographically organized. On the basis of these pointto-point relations in the projections from the cerebral cortex to the basal ganglia, the intrinsic basal ganglia connections and the output from the basal ganglia to the thalamocortical system, the existence of parallel, functionally segregated basal ganglia-thalamocortical circuits has been put forward (Alexander et al., 1986; Groenewegen et al., 1990). All thalamocortical circuits, via different thalamic nuclei, are directed to different areas of the frontal lobe, in this way emphasizing the importance of the basal ganglia for the wide array of motor, executive, and emotional-motivational functions subserved by the frontal lobe, which includes primary motor, premotor, and associative (cognitive) and 'limbic' prefrontal cortical areas.

\section{Convergence and segregation of information}

Next to a parallel arrangement of connections within the basal ganglia and the basal gangliathalamocortical circuitry, convergence of information at several levels is likewise characteristic for the organization of basal ganglia circuits and essential for the understanding of basal ganglia functioning. For example, at the level of the striatum, intricate patterns of overlap and segregation exist between the afferents from different cortical and subcortical sources. Because the medium-sized striatal output neurons, due to their electrophysiological membrane properties (see above), are difficult to excite and need strong convergent input from excitatory inputs to become active, they are excellent 'coincident detectors' (Fig. 1; Houk et al., 1995). Thus, primarily on the basis of 'coincident' activity of different excitatory inputs-which may be derived from different cerebral cortical areas, midline- or intralaminar thalamic nuclei, the amygdala, or hippocampusspecific striatal neuronal populations can become active and produce a specific pattern of output through direct and/or indirect pathways. The spatial and temporal aspects of this coincident afferent activity determines the location and the identity of the striatal population ('ensemble'; Pennartz et al., 1994) that becomes active at a particular moment. At the level of the striatum, individual cortical areas can have multiple (small) areas of termination and provide in this way a mechanism through which various different combinations between afferent inputs can be established (Flaherty \& Graybiel, 1991). In more general functional terms, such arrangements are pre-eminently suited for the activation of a particular output on the basis of a specific set of inputs, namely, the detection of a particular sensory, motor, cognitive, or emotional context.

The integration of different streams of information can play a role not only in the striatum but also in other basal ganglia structures-namely, the pallidum, the substantia nigra, and the subthalamic nucleus. In particular the substantia nigra has been proposed to play an important role in integrating functionally different streams of information that influence motor and behavioral output (Haber et al., 2000). Nevertheless, the striatum can be considered the main locus of integrative aspects of basal ganglia functioning.

\section{FUNCTIONAL ASPECTS OF THE BASAL GANGLIA}

In the preceding paragraphs, I have emphasized, based on the functional-anatomical organization of the basal ganglia, that these brain structures play a role in a wide array of frontal lobe functions, ranging from sensorimotor and cognitive to emotional-motivational behavioral functions. Yet, the specific contribution of the 
basal ganglia to these functions has not been discussed. Most considerations on the functional role of the basal ganglia refer to the functional deficits that are caused by lesions of these structures, either 'natural' in the course of a disease or experimentally induced. From the extensive body of literature on this subject, we can conclude that such lesions lead either to a paucity and/or slowness of movements (hypo- and bradykinetic movement disorders) or to the release of unintentional movements, including choreatic, athetotic, dyskinetic, and dystonic movements (hyperkinetic movement disorders) (DeLong, 1990; Marsden \& Obeso, 1994). Globally considered, the hypo- and hyperactivity in the expression of basal ganglia disorders might be understandable from the above-discussed arrangement of direct and indirect pathways within the basal ganglia circuitry. An overactivity of the direct pathway relative to the indirect pathway would lead to a disinhibition of the thalamocortical system and thereby the uncoordinated or unsupervised release of motor output or cognitive processing. By contrast, the relative overactivity of the indirect pathway or network would ultimately lead to an overinhibition of the thalamocortical system, in this way preventing or impeding motor output or cognitive processing (DeLong, 1990; Gerfen \& Wilson, 1996). Also widely accepted, however, is that the basal ganglia are not crucial for the initiation of movements. This conclusion is based mainly on the observations that the electrophysiological activity of basal ganglia structures occurs relatively late in the initiation phase of a movement (Mink, 1996). Thus, while intentional movements can still be executed, even when the functions of the basal ganglia are impaired, these structures appear to be important for the quality and correctness of a movement or a behavioral act.

Therefore, the question of what the basal ganglia exactly contribute to the execution of movements and behavioral acts still remains open.
Interpretations based on lesions or interferences with, usually extensive parts of basal ganglia structures, might not reveal the real functional role of the basal ganglia under physiological conditions. Several hypotheses have been put forward, none of which has been definitely established. Thus, earlier hypotheses have entertained the idea that the basal ganglia are responsible for the automatic execution of learned movement sequences (Marsden, 1987; Marsden \& Obeso, 1994). Whether the basal ganglia are the site of 'storage' of motor programs or whether these programs are laid down in the cerebral cortex remained open, whereas the basal ganglia are considered crucial for 'calling up' these programs and the switching between them. Hikosaka (1994) suggested that the basal ganglia are responsible for the suppression and release mechanisms on such innate movements as locomotion, mastication, and so on via descending projections to the brain stem, as well as for similar mechanisms on complex, learned movements via the projections to the thalamocortical system. Hikosaka (1994) also proposed a role for the basal ganglia in the learning phase of complex movements, specifically also for the dopaminergic system and in his proposal, motor programs are 'stored' in the cerebral cortex. Following the phase of learning of motor programs, the basal ganglia would have a role only in the 'activation' of these programs, or in elements of those, in a particular context.

A recent hypothesis (Mink, 1996), taking into account the detailed knowledge of the basal ganglia circuitry outlined above, is more specific about the role of the basal ganglia in motor behavior. This hypothesis states that the basal ganglia are crucial for the facilitation of desired movements and the suppression of unwanted, competing movements (Mink, 1996). The basal ganglia, with their strong and tonic inhibitory input on the thalamic and mesencephalic target areas, act as a general brake on the expression of motor and behavioral output. At the moment that it is 'decided' in the prefrontal and 
premotor cortices to execute a motor program, this information is sent to the striatum, as well as in a corollary manner to the subthalamic nucleus. The activation of striatal neurons that give rise to the direct output pathway to either the internal segment of the globus pallidus or to the substantia nigra pars reticulata leads to a disinhibition of the thalamocortical system that, in turn, provides the final output for the desired movement (Fig. 4). The 'parallel' cortical excitation of the subthalamic nucleus leads to a higher activity of the basal ganglia output neurons not concerned with the intended movement or complex of movements, resulting in the suppression of potentially competing motor output (Mink, 1996). Future experiments will have to test this attractive hypothesis for its validity.

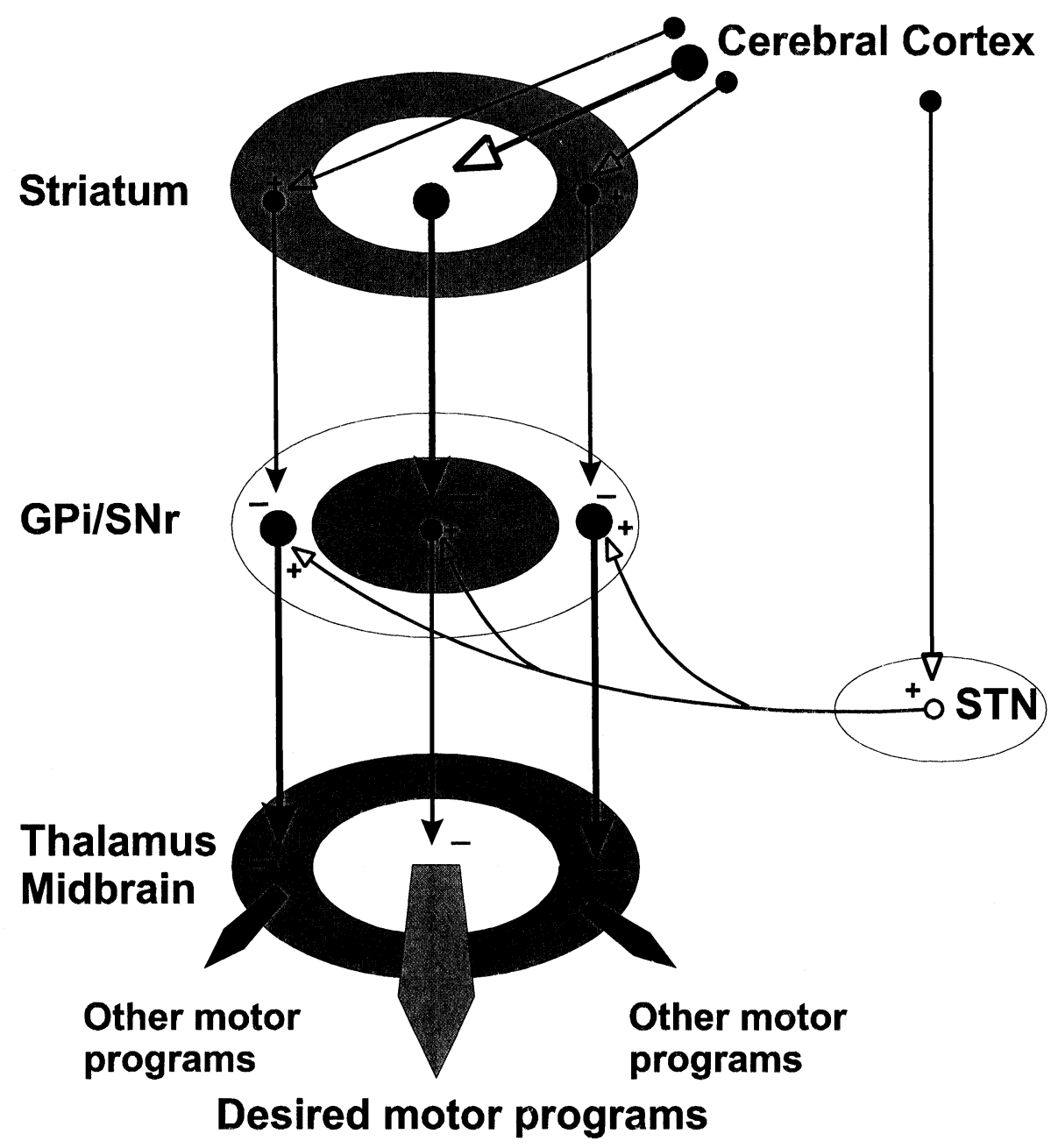

Fig. 4: Schematic representation of the interactions between the cortex cerebri, the striatum, the internal segment of the globus pallidus (GPi) or the reticular part of the substantia nigra ( $\mathrm{SNr}$ ) and the subthalamic nucleus (STN) that are hypothesized to lead to the selection of desired motor programs while suppressing other motor programs. Lines representing GABAergic projections have solid arrowheads, those using excitatory neurotransmitters have open arrowheads. Slightly adapted from figure 14 in Mink (1996). 


\section{Possible, different roles for dopamine}

As indicated above, levels of dopamine in the striatum determine, via their actions on dopamine D1 and D2 receptors, the output activity of the basal ganglia. In general terms, low levels of dopamine cause a strong inhibitory output of the basal ganglia to the thalamocortical system and to the brain stem. Low striatal dopamine levels are, therefore, associated with a paucity of movements as well as with cognitive and emotional/ motivational behavior. By contrast, high levels of striatal dopamine result in a low activity of the inhibitory basal ganglia outputs and, consequently, a 'disinhibition' of its targets. This situation is correlated with a facilitation of movements and cognitive/behavioral acts. In this way, dopamine appears to have a 'gating role' at the level of the striatum. This aspect of the role of dopamine in the basal ganglia is most probably correlated with variations in the tonic, relatively low rate of firing of dopamine neurons (Gerfen \& Wilson, 1996; Schultz, 2002).

Another, more differentiated role for dopamine has been hypothesized in the realm of the learning of movements and behavioral acts. On the basis of behavioral studies combined with in vivo electrophysiology of the dopaminergic system, Schultz and colleagues (for review, see Schultz, 2002) elucidated important aspects of the role of dopamine in guiding behavior in primates. Dopaminergic neurons in the ventral mesencephalon show phasic activations following the encounter of the animal with novel stimuli, particularly in relation to the presentation of primary reward. The phasic activations occur between 100 and $300 \mathrm{msec}$ following the stimulus and are short-lasting. Neurons in the medial substantia nigra pars compacta and the VTA, projecting to ventral striatal regions, tend to show a stronger activation than those in more lateral parts of the substantia nigra. Furthermore, in well- controlled experimental tasks of behavioral learning, such as Pavlovian or instrumental learning, the phasic activity of the dopaminergic neurons gradually shifts from the primary reward to conditioned stimuli that predict the primary reward. Interestingly, dopamine neurons show a depression in their activity if an expected reward, predictive on the basis of the previous learning process, does not appear (Schultz, 1998). Noteworthy is that the activations of the dopamine neurons are not related to any aspect of the movements that animals have to make during the tasks underlying the behavioral learning (Schultz, 2002).

The effect of a brief, phasic activation of the dopaminergic system, which at the striatal level leads to a spatially rather general release of dopamine, can be interpreted as follows. Dopamine terminals are present on striatal medium-sized spiny neurons and, at the ultrastructural level, terminate very close to the corticostriatal terminals on the spines of these neurons (Fig. 1; Smith \& Bolam, 1990). As discussed above, the cortico-striatal system is very precisely organized, and fibers from different although functionally related cortical areas converge on the same neuron, or on small groups of neurons ('ensembles'). These neurons will be activated only under specific circumstances, namely when a sufficient number of their excitatory corticostriatal afferents are active. Striatal mediumsized spiny neurons are therefore thought to be ideal 'context detectors' (Houk et al., 1995). The effect of the release of dopamine under these circumstances might be twofold.

- First, dopamine release might have an immediate focusing or attentional effect that could enable or facilitate the output of a particular ensemble of striatal neurons at the expense of other striatal outputs. In this way, dopamine, on the basis of rewarding or error signals, could have a gating or 'instructional' role. Such processes could contribute to the 
selection mechanisms that are thought to play a role at the level of the striatum (Pennartz et al., 1994; Redgrave et al., 1999).

- Second, dopamine may lead to plastic synaptic changes, building and shaping striatal output modules that, on the basis of a learning process, will be selectively and preferentially active in a particular context. In this way, dopamine would have a transient role in the process of reward-based motor and behavioral learning (Hikosaka, 1994; Houk et al., 1995; Schultz, 2002).

\section{CONCLUSIONS}

As discussed above, the basal ganglia appear to have a role in a wide range of sensorimotor, cognitive, and behavioral processes that are closely associated with the executive and motor functions of the (pre)frontal cortex. The selection of motor or behavioral programs, or elements thereof, appropriate for a particular context, might be one of the primary functions of the basal ganglia (Mink, 1996; Redgrave et al., 1999). Plasticity in the basal ganglia circuitry and learning processes are important fundaments for these functions. In particular, the ventral striatum might be crucial for the learning and execution of reward-related behavior, whereas the dorsal striatum is important for stimulus-response behavior (habits).

The development of the human basal ganglia, in particular the dopaminergic system, takes place over an extended period of time and most probably includes at least the first three decades of life (Segawa, 2000). The activity of tyrosine hydroxylase, as a marker for the dopaminergic system, and the expression of dopamine receptors vary significantly in this extended age period. Certain variables may reach 'adult' levels only in the fourth decade. The effects of lesions of the basal ganglia are also dependent upon the age at which they occur. Thus, whereas lesions of the dopaminergic system in early life lead to dystonic symptoms, after the third decade such lesions rather result in parkinsonistic symptoms (Segawa, 2000). Another disease in which the basal ganglia are most likely affected and with onset in childhood is Tourette's syndrome. This syndrome is characterized by multiple chronic tics that may be considered as the expression of unwanted, stereotyped movements or (fragments of) behavioral acts. Within the context of the hypothesis of Mink (1996), namely, that the basal ganglia play an important role in the release of desired and in the suppression of unwanted movements, the symptoms in Tourette's syndrome can be interpreted as the result of a defective suppression mechanism in the basal ganglia (Mink, 2001).

These two examples of basal ganglia disorders occurring in childhood illustrate different aspects of the role of the basal ganglia in the control of movements. In dystonia-namely, focal or more generalized prolonged contraction of (groups of) muscles leading to a disturbed posture-normal skillful movements are hampered by the unwanted contractions. In Tourette's syndrome, fragments of in principal normal movements are expressed beyond the control of the patient. In both cases, however, the mechanism to select a movement or a motor program is defective.

The question whether clumsiness, at least in part, may be attributed to a defective role of the basal ganglia in the process of the learning and execution of skillful movements is at present difficult to answer. It seems very likely that disturbances in the development of the cerebellum play an important role in clumsiness (Gramsbergen, 2003). Clumsiness, however, is a very heterogeneous concept in which various different aspects of motor control, including sensory feedback, can be involved. As far as deficient motor programming is involved, the basal ganglia probably play a role (see above). When 
inappropriate timing of contractions 'within' a more global motor program is affected, the cerebellum can be more involved. Both the basal ganglia and the cerebellum undergo an extended period of postnatal development, in humans spanning almost two decades. Factors that affect the normal development of the brain in this extended developmental period can affect the functions of both structures. An important issue for future research might be the more precise determination of the relative contribution of the basal ganglia and the cerebellum to the learning of skillful movements. Clearly both structures contribute to motor skill learning (Hikosaka et al., 2002), but the relative contribution of different brain structures remains to be elucidated. Although the roles of the cerebellum and the basal ganglia may be largely complementary, an interesting question is to what extent interactions between these structures, for example at the level of the brain stem or the thalamus, are contributing to the learning of skillful movements (Stein and Aziz, 1999; Doya, 2000; Hikosaka et al., 2002). Hopefully, this approach will provide more insight into the deficits in the central control of movements that are associated with clumsiness.

\section{REFERENCES}

Alexander GE, DeLong MR, Strick PL. 1986. Parallel organization of functionally segregated circuits linking basal ganglia and cortex. Ann Rev Neurosci 9: 357-381.

Alexander GE, DeLong MR, Strick PL. 1986. Parallel organization of functionally segregated circuits linking basal ganglia and cortex. Ann Rev Neurosci 9: 357-381.

Bolam JP, Hanley, JJ, Booth PAC, Bevan MD. 2000. Synaptic organisation of the basal ganglia. J Anat 196: 527-542.

Chevalier G, Deniau JM. 1990. Disinhibition as a basic process in the expression of striatal functions. Trends Neurosci 13: 277-280.
DeLong MR. 1990. Primate models of movement disorders of basal ganglia origin. Trends Neurosci 13: $281-285$.

Diaz J, Levesque D, Lammers CH, Griffon N, Martres MP, Schwartz JC, Sokoloff P. 1995. Phenotypical characterization of neurons expressing the dopamine D3 receptor in the rat brain. Neuroscience 65: 731-745

Doya K. 2000. Complementary roles of basal ganglia and cerebellum in learning and motor control. Curr Opin Neurobiol 10: 732-739.

Feger J, Bevan M, Crossman AR 1994. The projections from the parafascicular thalamic nucleus to the subthalamic nucleus and the striatum arise from separate neuronal populations: a comparison with the corticostriatal and corticosubthalamic efferents in a retrograde fluorescent double-labeling study, Neuroscience 60: 125-132.

Flaherty AW, Graybiel AM. 1991. Corticostriatal transformations in the primate somatosensory system. Projections from physiologically mapped body-part representations. J Neurophysiol 66: 1249-1263.

Gerfen CR, Wilson CJ. 1996. The basal ganglia. In: Swanson LW, Björklund A, Hökfelt T, eds, Handbook of Chemical Neuroanatomy, Vol. 12. Integrated Systems of the CNS. Part III. Amsterdam, The Netherlands: Elsevier; 371-468.

Gramsbergen AA. 2003. Clumsiness and disturbed cerebellar development: Insights from animal experiments. Neural Plast 10: 00-00.

Groenewegen HJ, Berendse HW, Wolters JG, Lohman AHM. 1990. The anatomical relationship of the prefrontal cortex with the striatopallidal system, the thalamus and the amygdala: evidence for a parallel organization. Prog Brain Res 85: 95-118.

Groenewegen HJ, Wright CI and Beijer AVJ. 1996. The nucleus accumbens: gateway for limbic structures to reach the motor system? In: Holstege G, Bandler R, Saper CB, eds, The Emotional Motor System. Prog Brain Res 107: 485-511.

Haber SN, Fudge JL, McFarland NR. 2000. Striatonigrostriatal pathways in primates form an ascending spiral from the shell to the dorsolateral striatum. J Neurosci 20: 2369-2382.

Hadders-Algra, M. 2003. Developmental coordination disorder: is clumsy motor behavior caused by a lesion of the brain at early age. Neural Plasticity 10: 39-50. 
Hikosaka O. 1994. Role of basal ganglia in control of innate movements, learned behavior and cognition-a hypothesis. In: Percheron G, McKenzie JS, Feger J, eds, The Basal Ganglia. IV. New Ideas and Data on Structure and Function. New York, NY, USA: Plenum Press; 589-596.

Hikosaka O, Nakamura K, Sakai K, Nakahara H. 2002. Central mechanisms of motor skill learning. Curr Opin Neurobiol 12: 217-222.

Houk JC, Adams JL, Barto AG. 1995. A model how the basal ganglia generate and use neural signals that predict reinforcement. In: Houk JC, Davis JL, Beiser DG, eds, Models of Information Processing In the Basal Ganglia. Cambridge, Massachusetts, USA: MIT Press; 117-130.

Marsden CD. 1987. What do the basal ganglia tell premotor cortical areas? Ciba Found Symp 132: 282-300.

Marsden CD, Obeso JA. 1994. The functions of the basal ganglia and the paradox of stereotaxic surgery in Parkinson's disease. Brain 117: 877-897.

Mink JW. 1996. The basal ganglia: Focused selection and inhibition of competing motor programs. Prog Neurobiol 50: 381-425.

Mink JW. 2001. Basal ganglia dysfunction in Tourette's syndrome: a new hypothesis. Pediatr Neurol 25: 190-198.
Parent A, Hazrati L-N. 1995. Functional anatomy of the basal ganglia. I. The cortico-basal gangliathalamo-cortical loop. Brain Res Rev 20: 91-127.

Pennartz CMA, Groenewegen HJ and Lopes da Silva FH. 1994. The nucleus accumbens as a complex of functionally distinct neuronal ensembles: An integration of behavioural, electrophysiological and anatomical data. Prog Neurobiol 42: 719761.

Redgrave P, Prescott TJ, Gurney K. 1999. The basal ganglia: a vertebrate solution to the selection problem? Neuroscience 89: 1009-1023.

Schultz W. 1998. Predictive reward signal of dopamine neurons. J Neurophysiol 80: 1-27.

Schultz W. 2002. Getting formal with dopamine and reward. Neuron 36: 241--263.

Segawa M. 2000. Development of the nigrostriatal dopamine neuron and the pathways in the basal ganglia. Brain Dev 22: S1-S4.

Smith AD, Bolam JP. 1990. The neural network of the basal ganglia as revealed by the study of synaptic connections of identified neurones. Trends Neurosci 13: 259-265.

Stein JF, Aziz TZ. 1999. Does imbalance between basal ganglia and cerebellar outputs cause movement disorders. Curr Opin Neurol 12: 667-669.

Wise SP, Murray EA, Gerfen CR. 1996. The frontal cortex-basal ganglia system in primates. Crit Rev Neurobiol 10: 317-356. 

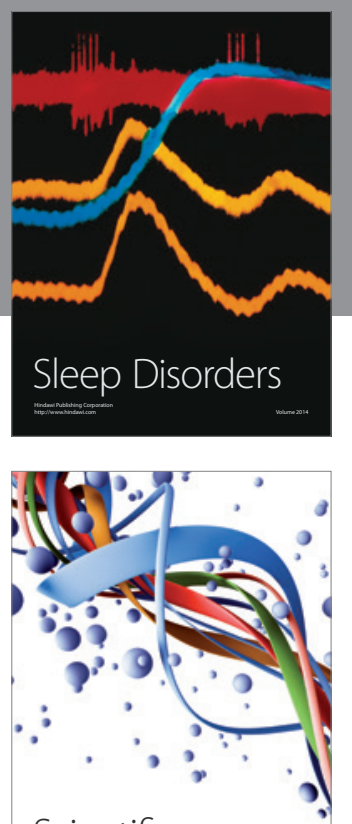

Scientifica
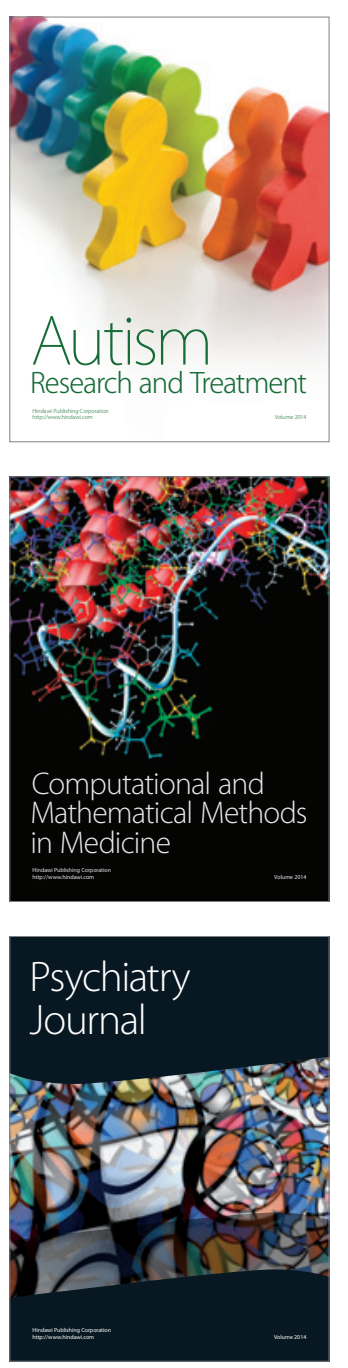
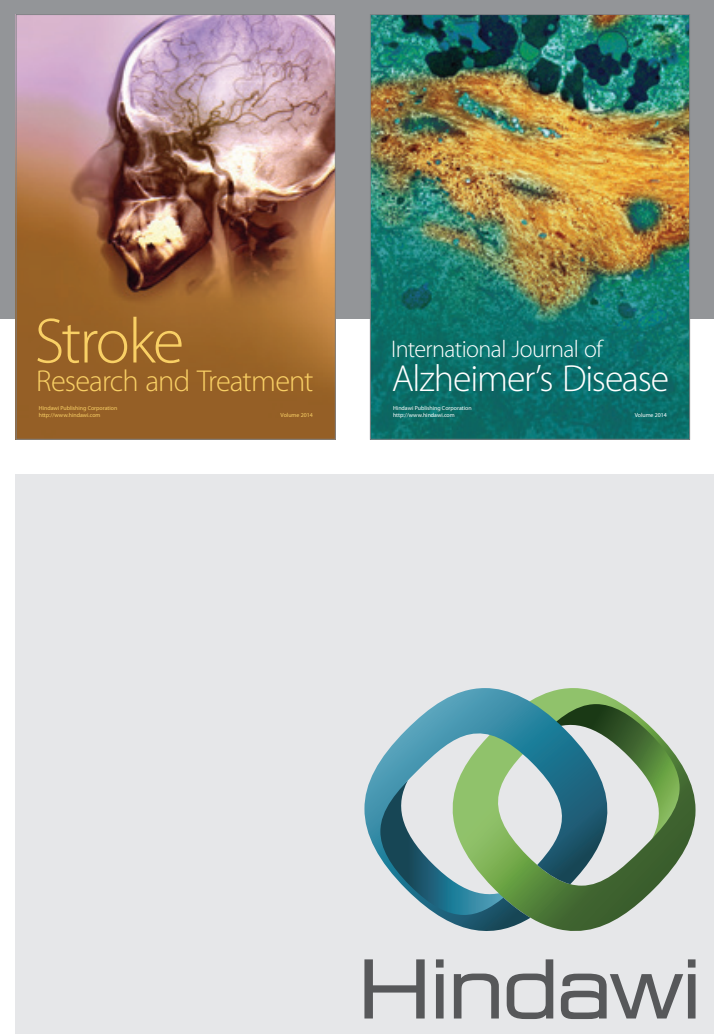

Submit your manuscripts at

http://www.hindawi.com
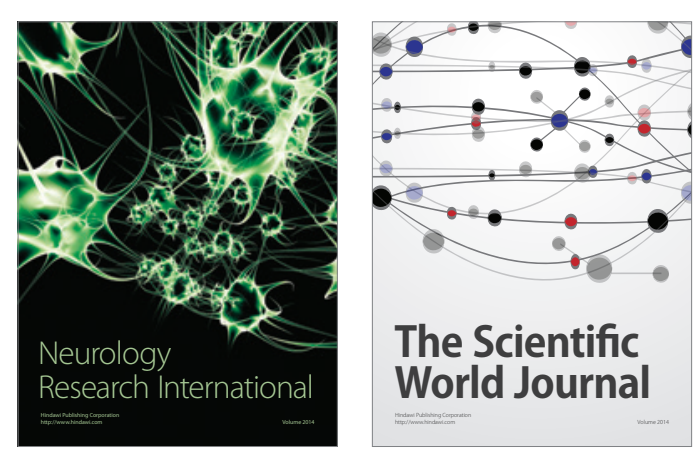

The Scientific World Journal

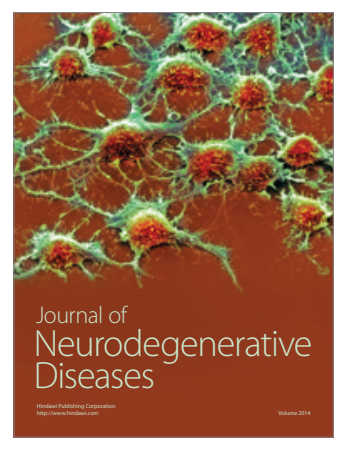

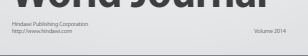

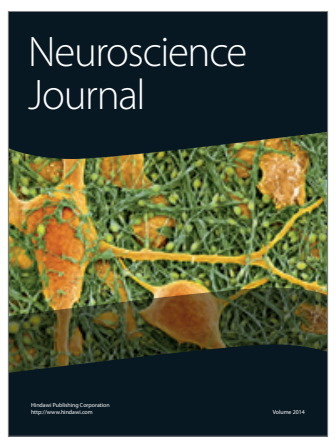

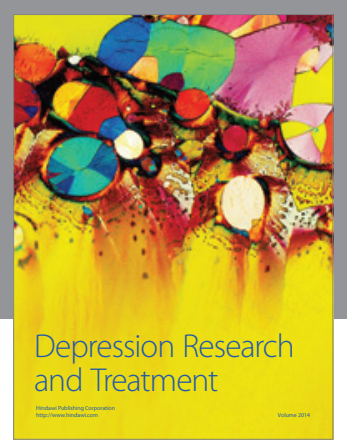
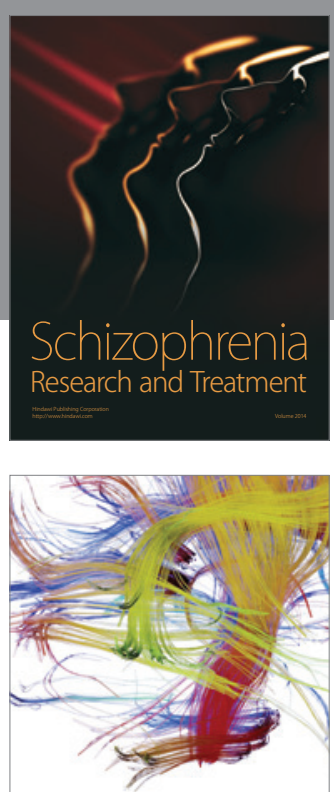

Brain Science

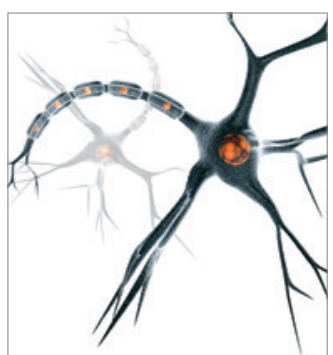

Neural Plasticity
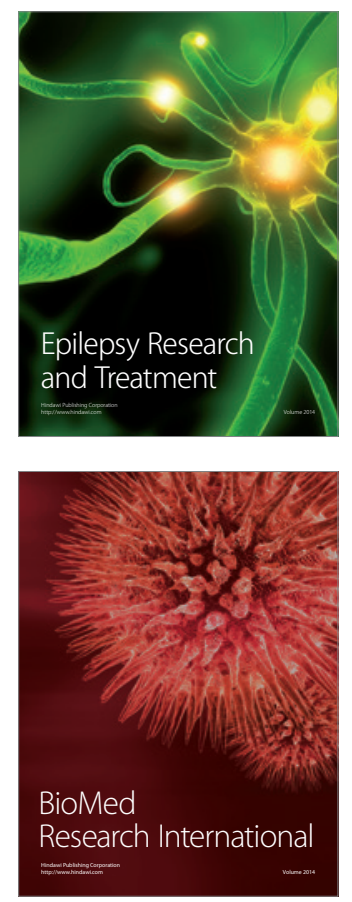

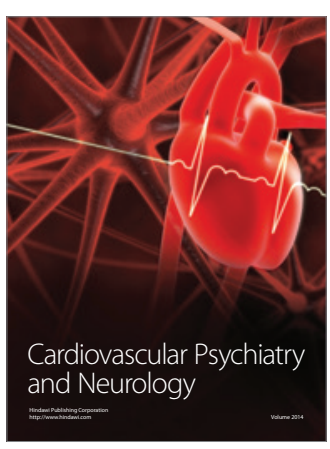

Parkinson's

Disease
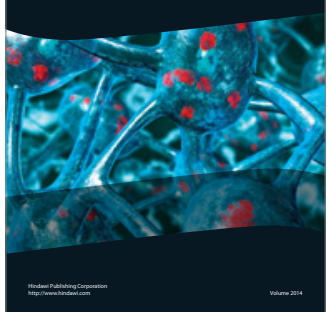